\title{
Validation of the ITER CXRS design by tests on TEXTORa)
}

R. J. E. Jaspers' , M. G. von Hellermann, E. Delabie, W. Biel, O. Marchuk, and L. Yao

Citation: Rev. Sci. Instrum. 79, 10F526 (2008); doi: 10.1063/1.2979874

View online: http://dx.doi.org/10.1063/1.2979874

View Table of Contents: http://aip.scitation.org/toc/rsi/79/10

Published by the American Institute of Physics

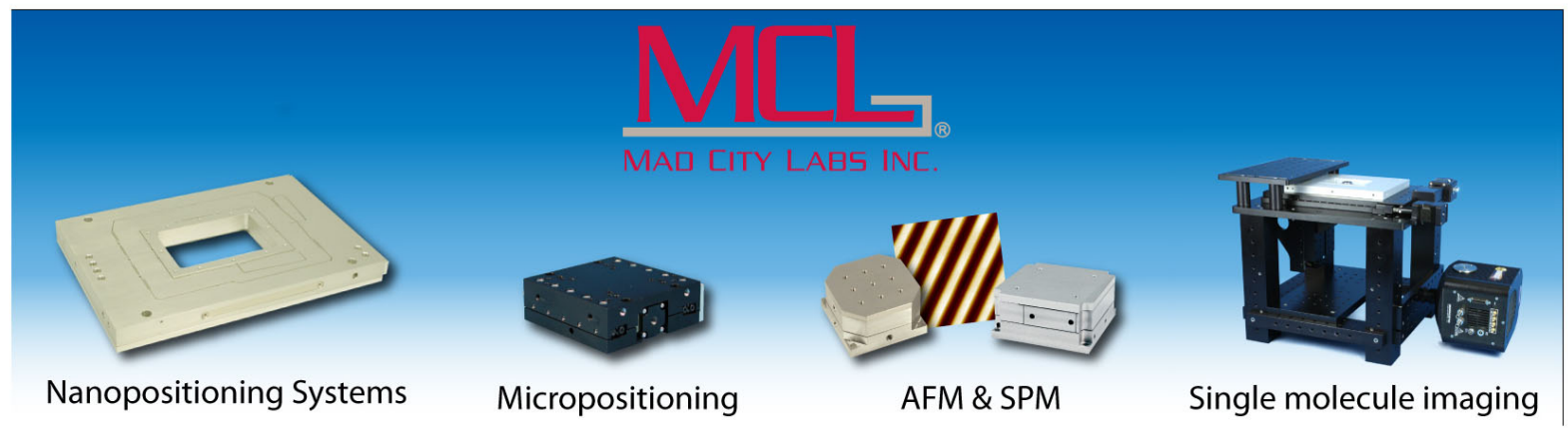




\title{
Validation of the ITER CXRS design by tests on TEXTOR ${ }^{\mathbf{a})}$
}

\author{
R. J. E. Jaspers, ${ }^{1, b)}$ M. G. von Hellermann, ${ }^{1}$ E. Delabie, ${ }^{1}$ W. Biel, ${ }^{2}$ O. Marchuk, ${ }^{2}$ and \\ L. $\mathrm{YaO}^{3}$ \\ ${ }^{1}$ FOM-Rijnhuizen, EURATOM-FOM, The Netherlands and Partner of the Trilateral Euregio Cluster (TEC), \\ P. O. Box 1207, Nieuwegein 3430 BE, The Netherlands \\ ${ }^{2}$ Forschungszentrum Jülich D-52428, EURATOM-FZJ, Germany \\ ${ }^{3}$ School of Physical Electronics, University of Electronic Science and Technology of China, Chengdu 610054, \\ People's Republic of China
}

(Presented 15 May 2008; received 9 May 2008; accepted 11 August 2008; published online 31 October 2008)

\begin{abstract}
The charge exchange recombination spectroscopy system (CXRS) for ITER is designed to measure the core helium concentration, and in addition, profiles of ion temperature and rotation. This highly demanding task, due to the huge background radiation (bremsstrahlung) and the high attenuation of the dedicated diagnostic neutral beam, requires high throughput spectrometers with high resolution. On TEXTOR, a CXRS system has been developed with the aim to test the physics implications of these specifications. (i) A relevant spectrometer has been tested. (ii) A method to determine the helium concentrations from the CXRS intensity, using the beam emission has been evaluated. A $20 \%$ discrepancy in beam emission was revealed. (iii) The determination of the magnetic pitch angle by the ratio of Balmer lines showed qualitatively the right behavior, although the accuracy was limited by the polarization sensitivity of the first mirror. (iv) The simulation code developed for the prediction of the CXRS spectra was quantitatively confronted with experimental data. (C) 2008 American Institute of Physics. [DOI: 10.1063/1.2979874]
\end{abstract}

\section{INTRODUCTION}

Over the past three decennia, the technique of charge exchange recombination spectroscopy (CXRS) has become an invaluable tool for diagnosing the ion temperature, plasma rotation, and impurity densities in tokamaks. ${ }^{1}$ The emission is based on the resonant charge exchange process between a fast neutral atom injected into the tokamak and the ions inside the hot plasma core. Such a CXRS diagnostic is also planned for ITER as being the principal measurement of the ion temperature profile and helium concentration. However, compared to present devices, the CXRS technique is more complicated on ITER due to (at least) two effects: (i) the attenuation of the neutral beam atoms is an order of magnitude higher and (ii) the background signal, dominated by the bremsstrahlung radiation, is even two orders of magnitude higher.

These effects determine largely the required specifications on the spectrometer: the etendue and the resolution. Moreover, the large beam attenuation also excludes the presently used technique of calculating the beam density by attenuation codes with sufficient accuracy. This problem might be circumvented by simultaneous recording of the beam emission signal to determine the impurity concentration.

Several aspects in the design of the ITER CXRS system lack a firm scientific basis and additional experimental validation is desirable. For this specific purpose on TEXTOR, a

\footnotetext{
a) Contributed paper, published as part of the Proceedings of the 17th Topical Conference on High-Temperature Plasma Diagnostics, Albuquerque, New Mexico, May 2008.

${ }^{b)}$ Electronic mail: r.j.e.jaspers@ @ijnhuizen.nl.
}

CXRS system has been built. This system has approximately the same geometry as on ITER, comprises of a spectrometer with characteristics close to the ITER requirements, and ample experimental time on TEXTOR is available for specific diagnostic tests. In this paper we report results of simulation validation, spectrometer performance, the use of the beam emission intensity, and the determination of the magnetic pitch angle from a spectral line ratio measurement.

\section{SIMULATION VALIDATION}

Starting point in the design of the ITER CXRS system is the performance prediction. For this purpose, a simulation code has been developed by Von Hellermann, ${ }^{2}$ predicting the CXRS signal for different devices and conditions. Based on this code the ITER CXRS system is being designed, and its performance is being checked. Moreover, this model forms also the basis for the Cramer Rao lower bound analysis used to make design decisions for the ITER CXRS spectrometer development. ${ }^{3,4}$ Inputs to this code are the plasma and beam parameters, geometry information, and detection information (spectrometer specification, transmission, and detector data). Based on this, the beam attenuation and the CXRS spectra are calculated, including the bremsstrahlung background, the passive emission, and the noise in the data. This allows quantifying the expected signal intensity as well as the statistical errors in the determination of impurity concentrations, temperature, rotation, etc.

Since the code includes many existing (JET, TEXTOR, Tore Supra, AUG, MAST, RFX) and planned (ITER, W7-X, HL-2A, EAST, SST) CXRS systems, an effort has been 


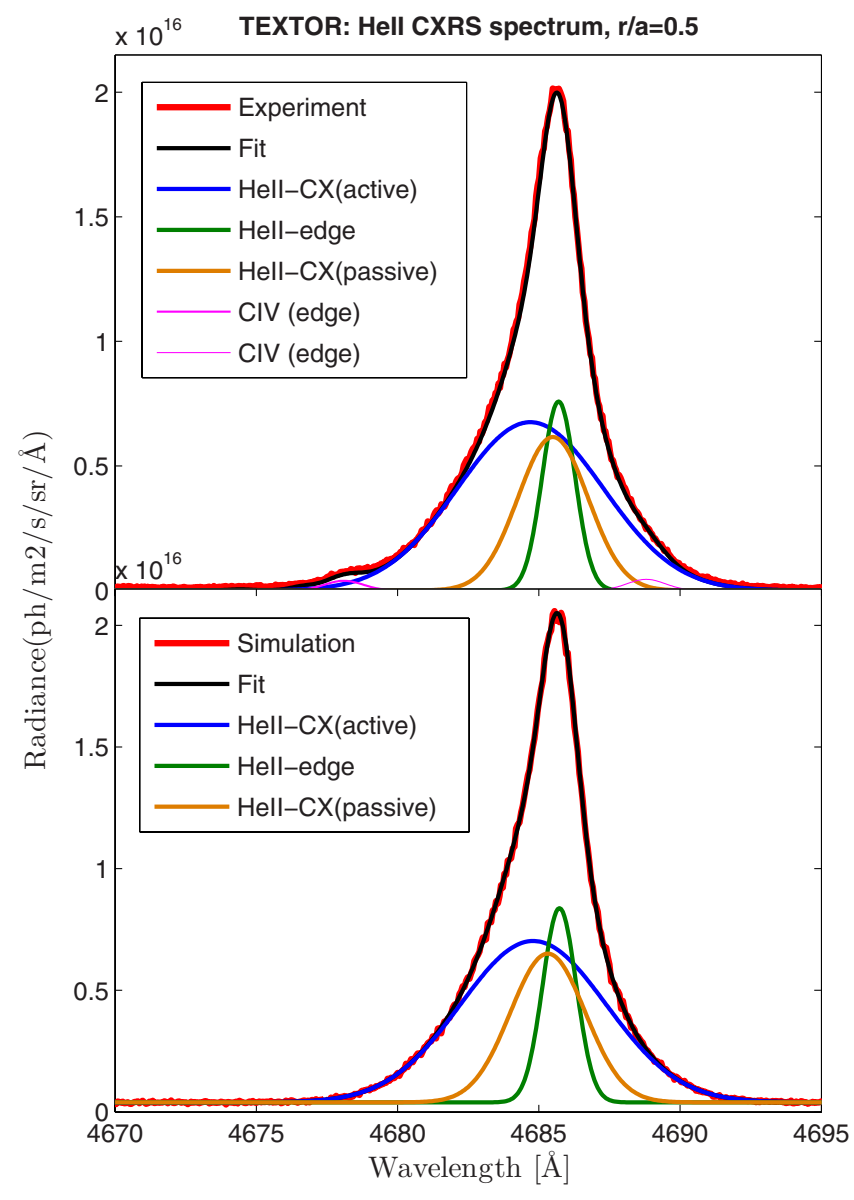

FIG. 1. (Color online) Top: measurement. Bottom: simulation. Shown here is a typical example of a helium spectrum of TEXTOR, compared with the results of the simulation code. In both cases the signal-to-noise ratio is comparable ( $\mathrm{S} / \mathrm{N}=35$ at half width).

started to compare the experimental results with the simulated ones and validate the code output in this way. As an initial result, Fig. 1 shows both the TEXTOR experimental and simulated results for a helium spectrum. The CXRS intensity and corresponding signal-to-noise ratio are comparable. Although this is not a final proof of the validity of the code, it shows that the principal assumptions are justified and correctly implemented. Lacking at the moment is a quantitative modeling of the passive emission, which (for the nonmodulated beam experiments) has a strong influence on the overall accuracy of the CXRS data. For the moment, just an ad hoc assumption on this is put into the code. Nevertheless, since ITER will operate with a modulated diagnostic neutral beam, the main result produced (i.e., to reach the required $10 \%$ accuracy in the core helium concentration, an etendue of $1 \mathrm{~mm}^{2} \mathrm{sr}$ for the spectrometer is required) seems justified. Further parameter scans on TEXTOR (and other devices) are planned in the near future to fully validate the code results.

\section{SPECTROMETER}

Based on the performance predictions for CXRS on ITER, a spectrometer with an etendue of $1 \mathrm{~mm}^{2} \mathrm{sr}$, a transmission of $50 \%$, and a linear spectral dispersion of about $0.25 \mathrm{~nm} / \mathrm{mm}$ is required. ${ }^{4}$ Such a system has been installed on TEXTOR. The spectrometer is designed by the Russian

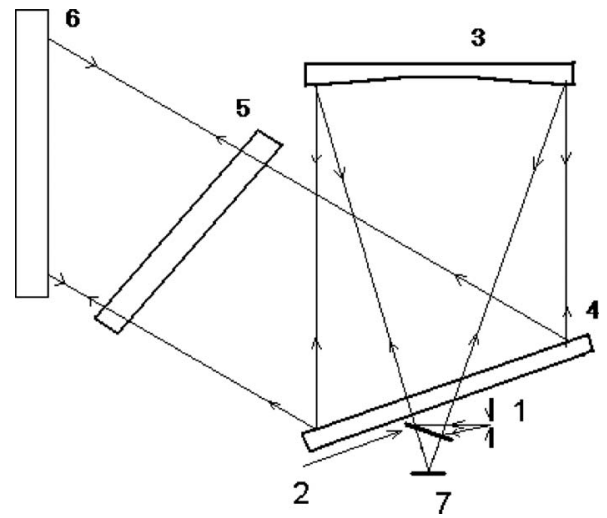

FIG. 2. High resolution high etendue echelle spectrometer, developed at TRINITI. Elements: $1=$ entrance slit, $2=$ small flat mirror, $3=$ spherical mirror, $4=$ flat mirror with hole, $5=$ correction element, $6=$ ruled grating, and $7=$ image plane.

TRINITI institute and is based on the Pfund (or Tarasov) scheme. The optical scheme is depicted in Fig. 2. The spectral instrument operates with 200 grooves $/ \mathrm{mm}$ echelle grating, has a $65^{\circ}$ blaze angle, and a maximum reflectivity efficiency of about $60 \%$. For He II $(468 \mathrm{~nm})$ it is used in 19th order, for C VI $(529 \mathrm{~nm})$ in 17 th order, and for $\mathrm{H}_{\alpha}(656 \mathrm{~nm})$ in 13th order. The overall transmission of the spectrometer was experimentally determined to be around $20 \%$. This relatively low value, resulting from the many surfaces, in combination with the difficult alignment procedure was regarded as the main shortcoming of this system. On the other hand, the high etendue, large dispersion, small spectral resolution, and excellent image quality still make it a useful instrument. For application on ITER, however, improved concepts are presently being investigated. ${ }^{5}$

\section{BEAM EMISSION SPECTROSCOPY}

The simultaneous recording of the beam emission (at the Balmer- $D_{\alpha}$ wavelength of $656.2 \mathrm{~nm}$ ) and the impurity CXRS spectra (for instance, carbon C VI or helium He II) have the potential advantage that the impurity concentration can be determined without the use of a beam attenuation code, which suffers from an exponentially increasing error with attenuation. ${ }^{6}$ Moreover, and at least equally advantageous, this method does not rely on an absolute calibration of the spectrometers. Only a relative sensitivity of both instruments suffices, which can be obtained from a comparison of the bremsstrahlung emission in the spectral background.

The ratio of both measurements $\left(I_{\mathrm{CXRS}}\right.$ and $\left.I_{\mathrm{BES}}\right)$ provides the impurity concentration, without the need to calculate the local neutral beam density, ${ }^{2}$

$$
\frac{n_{z}}{n_{e}}=\frac{I_{\mathrm{CXRS}}}{I_{\mathrm{BES}}} \frac{Q_{\mathrm{BES}}}{Q_{\mathrm{CXRS}}} .
$$

This method has two caveats. The atomic emission rates $Q_{\mathrm{CXRS}}$ and $Q_{\mathrm{BES}}$ do depend on the collision energy, i.e., beam energy $E_{\text {beam }}$, electron density $n_{e}$, plasma temperature $T_{i}$, and also impurity ion composition $n_{z}$. So the apparent simplicity of Eq. (1) hides in truth an iterative process. However, both for the beam-stopping calculation as well as for the ratio of $Q_{\mathrm{BES}} / Q_{\mathrm{CXRS}}$, the iteration usually takes only a 


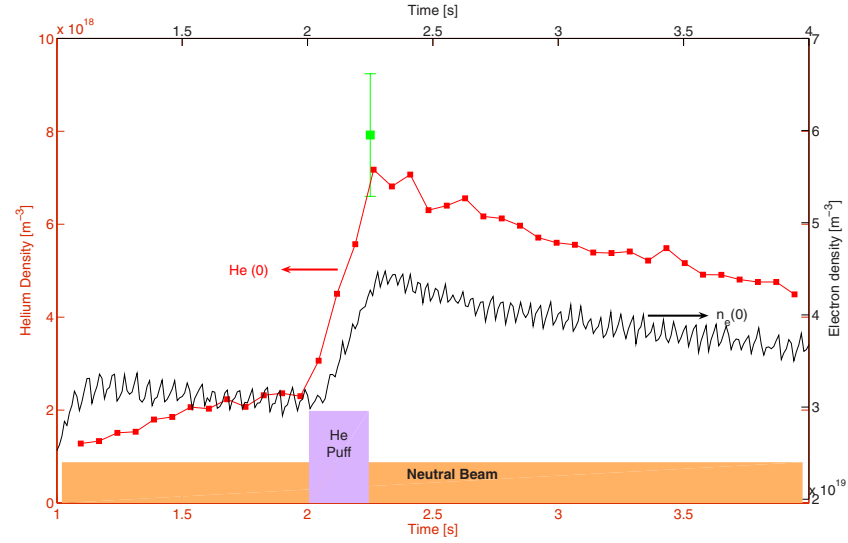

FIG. 3. (Color online) The helium density determined from the increase in the electron density after a puff (single data point), and from the ratio of CXRS and BES intensities (upper line with data points). Within the error bar, agreement is obtained. (The electron density is given by the lower line).

few steps and converges rapidly. The second caveat is the wavelength variation in the calibration factor for the case of the CXRS spectrum not being at the same wavelength as the Balmer- $H_{\alpha}$ spectrum. In this case, we can use the underlying bremsstrahlung as cross reference. (Note that reflections inside the vessel might complicate this approach). ${ }^{7}$

This method, i.e., determining the impurity content by the ratio of the CXRS and beam emission spectroscopy (BES) signals, has been tested in an experimental setup on TEXTOR. Here, the light from the plasma is guided by fibers to a beam splitter, which transmits the helium and carbon emission and reflects the $D_{\alpha}$ wavelength. To test the accuracy a small helium gas puff was introduced into the plasma. The rise in electron density could be fully attributed the helium increase, thereby providing immediately an absolute estimate of the helium concentration. That specific example is shown in Fig. 3 together with the density determined from the ratio CXRS/BES intensity. Within the error bars agreement is found. A direct comparison between the measured beam density (from the beam emission signal) and the calculated beam density (using the attenuation code CHEAP) ${ }^{2}$ revealed a discrepancy of about $20 \%$, the experimental values being lower than the calculated beam density. Whether this can be attributed to uncertainties in atomic cross sections is presently under study. ${ }^{8}$

\section{MOTIONAL STARK EFFECT}

Another application of the beam emission spectrum is given by analyzing the motional Stark effect, i.e., the spectral and polarization characteristics of the emission, introduced by the Lorentz electric field: $E_{L}=v_{\text {beam }} \times B$, with $v_{\text {beam }}$ being the beam velocity and $B$ the magnetic field. Whereas the direct measurement of the polarization has become the standard method on several devices, ${ }^{9}$ here investigated is the possibility that the pitch angle can also be obtained from the intensity line ratio of the two orthogonal components $I_{\pi}$ and $I_{\sigma}$,

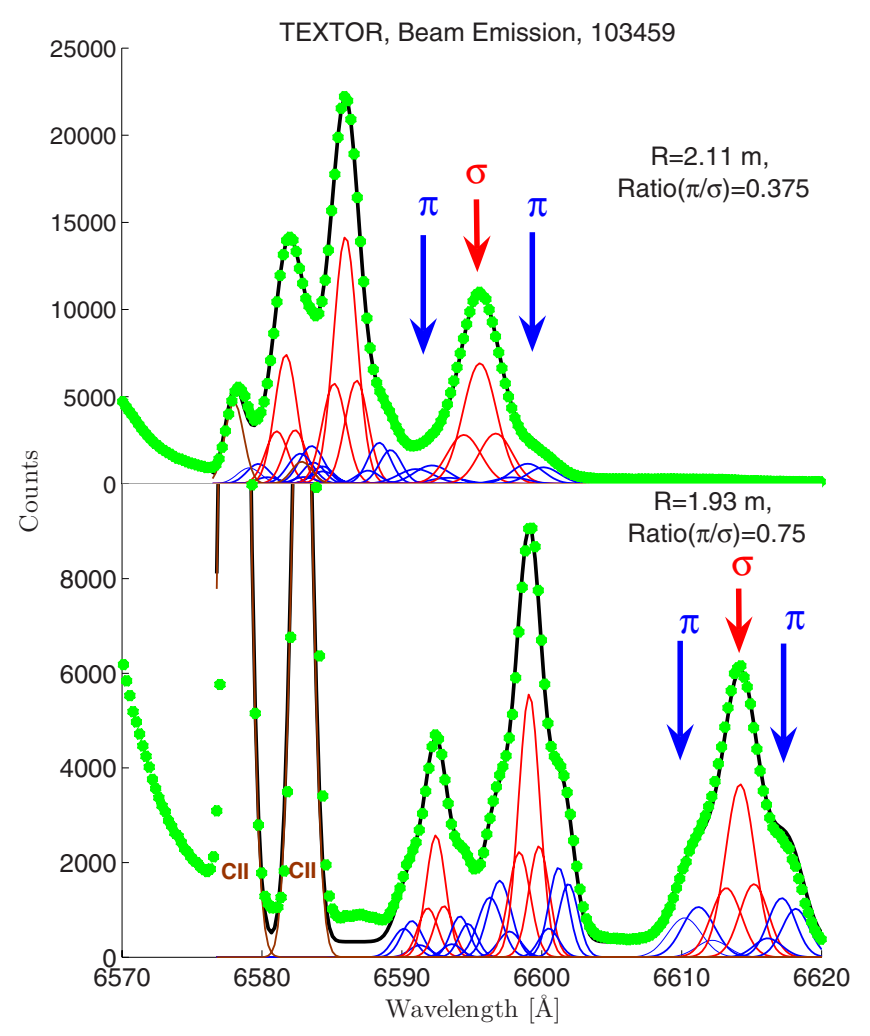

FIG. 4. (Color online) Measured beam emission at the edge (top) and in the center (bottom) of the plasma. A clear difference in the ratio between the $I_{\pi}$ and $I_{\sigma}$ intensity is observed.

$$
\frac{I_{\pi}}{I_{\sigma}}=\frac{\sin ^{2} \Theta}{1+\cos ^{2} \Theta}, \quad \Theta=\Theta_{0} \pm\left(\frac{r}{q R_{0}}\right),
$$

where $\Theta$ is the angle between the viewing line and the electric field vector, $\Theta_{0}$ is the angle in the case of no poloidal field, $r$ is the minor radius, $q$ is the safety factor, and $R_{0}$ is the major radius of the tokamak. So the ratio measurement allows to determine $\Theta$ and so the poloidal magnetic field (or $q$ ). To mimic the ITER situation, one should make sure that the angle $\Theta$ between line of sight and electric field are similar. For ITER, viewing the diagnostic neutral beam from the upper port $3, \Theta_{0}$ is in the range between $32^{\circ}$ and $43^{\circ}$ for observations between the core and the edge. For the setup at TEXTOR this amounts to $\Theta_{0}=52^{\circ}-63^{\circ}$. (Note: this has similar sensitivity as for ITER, being almost $\left.90-\Theta_{0, \text { ITER }}\right)$.

Two typical examples are shown in Fig. 4, one measured at the center of TEXTOR, corresponding to a large value for $\Theta$, and one at the edge where $\Theta$ is lower. Figure 5 compares the corresponding measured profiles (datapoints) of this line ratio with the expected one (full lines). The large disagreement can be explained by the reflectivity characteristics of the first metallic mirror. Taking into account the different reflections for parallel $(s)$ and perpendicular $(p)$ polarizations $\left(R_{p}=0.79\right.$ and $R_{s}=0.25$, respectively), the data are consistent (dashed lines). This extra correction and corresponding uncertainty in $R_{s}$ and $R_{p}$ however limits the accuracy of determining $q$ from the measurements.

Just to show the sensitivity of the line ratio, a few discharges were made with reversed plasma current. In that case, the sign in Eq. (2) changes, giving rise to an observable 


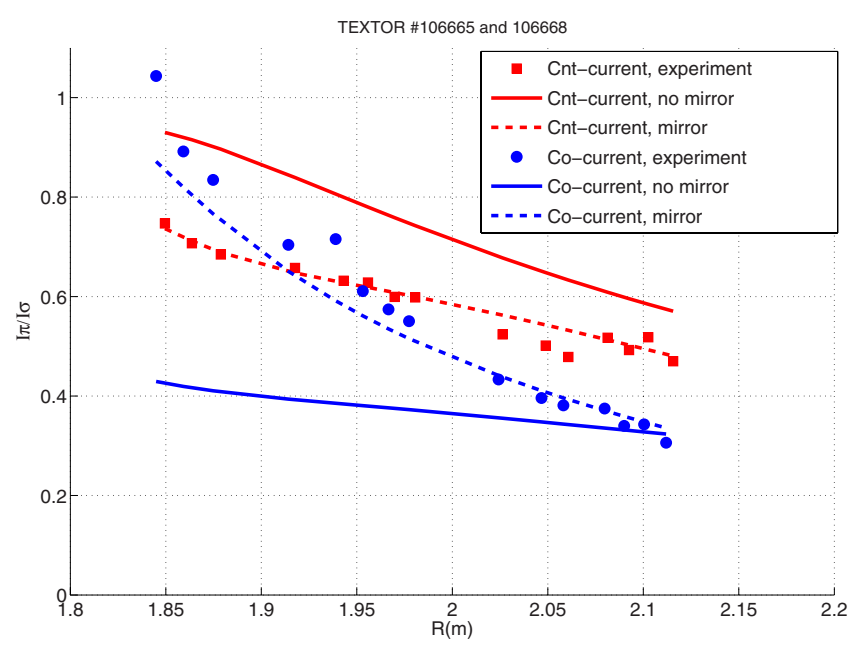

FIG. 5. (Color online) The line ratio for two case with opposite current [i.e., where the sign in Eq. (2) changes]. The effect of the mirror is to make the measurement less sensitive. Nevertheless, experimental data show the expected trend.

change in line ratio. This is shown by the red data in Fig. 5 and conforms with expectation, but due to the mirror reflection the sensitivity is strongly reduced.

\section{CONCLUSION}

The high etendue, high resolution spectrometer developed at the TRINITI institute and implemented on TEXTOR, operates according to specifications and improves the quality of the CXRS measurements considerably. Further development in optimizing the efficiency (the total spectrometer transmission is less than 25\%) and a more robust optical scheme (i.e., easier in alignment) are certainly advisable.

Notwithstanding these aspects, valuable results and tests for the development of the ITER CXRS system have been obtained,

- The comparison between the measured beam emission and the one calculated using a beam attenuation code revealed some $20 \%$ discrepancy, which deserves further attention.

- The ratio motional Stark effect (MSE) method to determine the magnetic pitch angle was hampered by the reflec- tivity characteristics of the first mirror for different polarizations. This effect could however be quantified and understood, but this method cannot yet compete with the standard approach of MSE: measuring the polarization of a single line directly. ${ }^{9}$ Since a high measurement accuracy $(<1 \%)$ of the line ratio is required for a reasonable calculation of the safety factor, more effort is required in optimizing and characterizing the first mirror.

- A simulation code for the prediction of the CXRS spectra has been developed and could now be quantitatively confronted with experimental data. First examples are encouraging. A more intensive comparison including different devices, different impurity lines, and different plasma parameters is planned to validate the ITER CXRS prediction.

\section{ACKNOWLEDGMENTS}

This work, supported by the European Communities under the Contract of Association between EURATOM-FOM, was carried out within the framework of the European Fusion Program. The views and opinions expressed herein do not necessarily reflect those of the European Commission.

${ }^{1}$ R. C. Isler, Plasma Phys. Controlled Fusion 36, 171 (1994).

${ }^{2}$ M. G. von Hellermann, E. Delabie, R. J. E. Jaspers, W. Biel, O. Marchuk, H. P. Summers, A. Whiteford, C. Giroud, N. C. Hawkes, and K. D. Zastrow, AIP Conf. Proc. 988, 165 (2008).

${ }^{3}$ J. F. F. Klinkhamer, N. C. J. van der Valk, M. G. von Hellermann, and R. J. E. Jaspers, Rev. Sci. Instrum. 79, 10F331 (2008).

${ }^{4}$ M. G. Von Hellermann, R. Jaspers, W. Biel, A. Litnovsky, O. Neubauer, M. Pap, N. C. Hawkes, C. Marren, B. Walton, Y. Kaschuck, V. Serov, S. Tugarinov, W. Vliegenthart, K. Moddemeijer, C. Walker, and C. Ingesson, Rev. Sci. Instrum. 77, 10F516 (2006).

${ }^{5}$ W. Biel, S. Brezinsek, R. Burhenn, E. Delabie, A. Greiche, W. von Hellermann, R. Jaspers, J. Koning, R. Koenig, F. Klinkhamer, H. G. Lee, O. Marchuk, and the TEXTOR team, Proceedings of the IAEA International Workshop on Challenges in Plasma Spectroscopy for Future Fusion Research Machines, Jaipur, India, February 2008 (unpublished), Vol. 1.

${ }^{6}$ W. Mandl, R. C. Wolf, M. G. von Hellermann, and H. P. Summers, Plasma Phys. Controlled Fusion 35, 1373 (1993).

${ }^{7}$ K. D. Zastrow, S. R. Keating, L. Marot, M. G. O'Mullane, G. de Temmerman, and JET-EFDA Contributors, Rev. Sci. Instrum. 79, 10F527 (2008).

${ }^{8}$ O. Marchuk, G. Bertschinger, W. Biel, D. Reiter, and the TEXTOR team, Rev. Sci. Instrum. 79, 10F532 (2008).

${ }^{9}$ F. M. Levinton, Rev. Sci. Instrum. 70, 810 (1999). 